To appear in the Journal of Geophysical Research.

\title{
A study of the propagation of Alfvén waves in the auroral density cavities
}

\author{
V. Génot, P. Louarn \\ Observatoire Midi-Pyrénées, Toulouse, France \\ D. Le Quéau \\ Centre d'Etude Spatiale des Rayonnements, Toulouse, France
}

\begin{abstract}
From the Viking and, more recently, the FAST spacecraft observations, it is known that the auroral acceleration regions correspond to relatively small scale density cavities (a few $\mathrm{km}$ to a few $10 \mathrm{~km}$ in a direction perpendicular to the magnetic field). In order to study how Alfvén waves can contribute to the auroral acceleration, we analyze their propagation in the presence of the sharp density gradients that characterize the edges of these cavities. It is shown that an Alfvén wave packet entering into the cavity quickly develops short perpendicular length scales in the region of density inhomogeneity. Transverse scales of the order of $c / \omega_{p e}$ are reached after a propagation of a few tenths of second, which corresponds to approximately $2000 \mathrm{~km}$ in the cavity. This contributes to the creation of significant parallel electric field in the region of density gradient. Its amplitude is enhanced by the formation, on the gradients, of strong space charges due to the ion polarization drift. As the auroral cavities are known to be strong current regions, the density gradients would thus be the sites of particularly powerful wave/particle energy transfer and consequently, if the incident flux of Alfvén waves coming from the magnetosphere is high enough, of the strongest particle acceleration.
\end{abstract}




\section{Introduction}

Many mechanisms have been proposed to explain the acceleration of particles in the auroral regions: the anomalous resistivity, the formation of double layers or electrostatic shocks, the direct acceleration by lower-hybrid waves and the interaction with kinetic (or inertial) Alfvén Waves (AW) (see [Borovsky, 1993] and references therein). However there is still no consensus concerning the identification of a dominant process and some of these different mechanisms could well act together, in a way not well understood, in order to produce the observed acceleration up to $10 \mathrm{keV}$ energies. The interest for the study of the contribution of the kinetic/inertial AW in the auroral acceleration has been recently renewed by observations of the Freja spacecraft. Using measurements obtained in the topside auroral ionosphere, it was shown that strong electromagnetic disturbances interpreted as being kinetic/inertial AW possibly play an important role in the wave-particles energy transfer [Louarn et al., 1994; Wahlund et al., 1994; Seyler et al., 1995; Volwerk et al., 1996; Bellan and Stasiewicz, 1998; Stasiewicz et al., 1998], a result that seems to be confirmed by the Interball auroral spacecraft. More precisely, the measured Poynting flux (up to a few $10^{-3} \mathrm{~W} / \mathrm{m}^{2}$ ) is typically of the order of the kinetic energy flux of precipitating particles, and then, large enough to significantly contribute to the acceleration if it is efficiently dissipated.

One of the theoretical bases of the AW acceleration model is a work by [Hasegawa, 1976] who showed that an AW propagating at a sufficiently large angle with respect to the magnetic field develops a finite parallel electric field. For low $\beta$ plasma, this regime is reached when the perpendicular wavelength $\left(k_{\perp}^{-1}\right)$ becomes of the order of the electron inertial length $\left(c / \omega_{p e}\right)$. As proposed by Goertz and Boswell, [1979] (see also the review by $[$ Lysak, 1990]), this property can be used for building non-ideal and time-dependent models of the magnetosphere-ionosphere coupling. On the experimental point of view Maggs and Morales [Maggs and Morales, 1996; Maggs and Morales, 1997] showed that filamentary structures (density depletion and/or temperature gradients) are the driving source for the magnetic and density fluctuations in the edge of the field-aligned striations which have length scales of the order of $c / \omega_{p e}$. These laboratory experiments thus suggest that the plasma inhomogeneities, in a direction perpendicular to the magnetic field, could play an important role in the AW dissipation. This has to be related to Viking and Fast spacecraft observa- tions that reveal the existence of deep plasma cavities (with a factor of the order of ten in the density variation) with a typical perpendicular scale of a few kilometers and where a very efficient acceleration takes place [Louarn et al., 1990; Hilgers et al., 1992; Ergun et al., 1998; Strangeway et al., 1998]. The existence of these small scale plasma cavities must be taken into account in any model of the auroral particle acceleration. We propose here a study of the AW propagation in a realistic model of these auroral cavity regions.

In order to estimate the contribution of the AW in the auroral acceleration the mechanism by which the perpendicular wavelength of the AW shrinks must be identified and the amplitude of the parallel electric field that develops during the propagation must be calculated. This problem has been specifically studied in a serie of papers by Streltsov and Lotko ( [Streltsov and Lotko, 1997] and references therein). These authors relate the existence of the small scale sheet-like structures that correspond to the discrete auroral arcs to the formation and evolution of dispersive field line resonance layers. This work is based on the analysis of the Alfvén wave propagation in the dipolar magnetic geometry and uses average, global scale model of the plasma distribution. The present analysis complements this work in the sense that we explicitly focus our study on the description of the Alfvén wave propagation in the strongly perturbed and inhomogeneous plasma that characterizes the auroral regions during phase of intense magnetospheric activity.

The physical view of the process we want to put in relief is the following: the propagation of the Alfvén wave induces a perpendicular motion of the ions due to the polarization drift (it is the origin of the perpendicular current that follows the Alfvén wave propagation). If the wave presents a finite perpendicular wave vector, this drift is not uniform, which leads to ion concentration and rarefaction and thus to the formation of space charges. When the finite electron mass is taken into account these space charges cannot be instantaneously compensated by equal electron density variations and a parallel electric field forms. This constitutes the classical inertial AW model of the auroral acceleration. In the presence of sharp perpendicular density gradients that are actually observed in the auroral regions, the ion polarization drift generates space charges whatever the Alfvén wave characteristics. The space charges will be compensated by the electron motion associated to the formation of a parallel electric field. This field, linked to the existence of the density gradients, could be larger than the one 
expected in the strict inertial AW model.

Instead of performing a Fourier analysis of the propagation equation, we will numerically model the propagation and the deformation of an Alfvénic pulse coming from the magnetosphere. This linear study could simulate the impulsive response of the auroral regions to a sudden influx of energy from the magnetosphere. We will address two precise questions: How is the AW pulse propagation modified by cavities? and, more specifically: To what extent do density cavities help incoming AW to develop a strong parallel electric field $(\mathrm{a}$ few $\mathrm{mV} / \mathrm{m})$ ?

\section{Equations of propagation}

The equations are similar to those of Goertz and Boswell, [1979] but include the perpendicular spatial dependence of the density and the effect of the magnetic field line convergence. We use a first order approximation of the dipolar magnetic field adapted to the study of high latitude phenomena taking place not too far from the Earth (less than $4 R_{e}$ ). Locally near the Earth one states $x \ll h$ and $y \ll h$ where $x$ and $y$ are the coordinates in the horizontal directions and $h=z+R_{e}$ is the distance from the Earth center. Thus at the first order in $x / h$ and $y / h$ the magnetic field components take the form: $B_{x} \propto 3 x / h^{4}, B_{y} \propto 3 y / h^{4}, B_{z} \propto 2 / h^{3}$, and we consider the system of natural curvilinear coordinates: $d s^{2}=h_{1}^{2} d x_{1}^{2}+h_{2}^{2} d x_{2}^{2}+h_{3}^{2} d x_{3}^{2}$ where $x_{3}$ is the abscissa along $B$ field line, $x_{1}$ is the coordinate in the perpendicular direction (North-South) and $x_{2}$ completes the system in the longitudinal direction. The metric factors are given by $h_{1}^{2}=h_{2}^{2}=B_{0} / B_{z}$ and $h_{3}=1$. $B_{0}$ is the ambient magnetic field for a reference altitude. In the following we note $\varphi^{2}=B_{0} / B_{z}$ and redefine $x=x_{1}, y=x_{2}$ and $z=x_{3}$. The reduction of the perpendicular length scale in the Earth direction is contained in the new definition of $x$ and $y$. We use the cold plasma approximation, which is justified given the very small $\beta$ of the auroral plasma (for instance at an altitude of $\left.5000 \mathrm{~km}, v_{A} \sim c / 3 \gg v_{t h}\right)$. We also assume $E_{y}=0$ and $\frac{\partial}{\partial y}=0$ (2D model). The transverse current is the polarization drift current and it is carried by the ions:

$$
j_{x}=\frac{m_{i} n_{i}}{B^{2}} \frac{\partial E_{x}}{\partial t}
$$

Eliminating $b_{y}$ and $j_{x}$ in the Maxwell equation system gives the first equation of the model:

$$
\frac{\partial^{2} E_{x}}{\partial t^{2}}=\frac{v_{A}^{2}}{\varphi} \frac{\partial}{\partial z}\left(\frac{\partial \varphi E_{x}}{\partial z}-\frac{\partial E_{z}}{\partial x}\right)
$$

$v_{A}=B / \sqrt{\mu_{0} n_{i} m_{i}}$ is the Alfvén velocity which is a function both of $z$ and $x$. The ion and electron motions are decoupled and the time derivatives of the conservation laws write respectively:

$\frac{\partial^{2} \delta n_{i}}{\partial t^{2}}=-\frac{1}{\varphi} \frac{\partial^{2}}{\partial x \partial t}\left(n v_{i x}\right)=-\frac{n m_{i}}{\varphi e B^{2}} \frac{\partial^{2}}{\partial t^{2}}\left(\frac{\partial E_{x}}{\partial x}+\frac{\partial \ln n}{\partial x} E_{x}\right)$

$$
\frac{\partial^{2} \delta n_{e}}{\partial t^{2}}=-\frac{1}{\varphi^{2}} \frac{\partial^{2}}{\partial z \partial t}\left(\varphi^{2} n v_{e z}\right)=\frac{e}{\varphi^{2} m_{e}} \frac{\partial \varphi^{2} n E_{z}}{\partial z}
$$

assuming at the first order $n_{e} \simeq n_{i} \simeq n$. Taking the second time derivative of Gauss' law and injecting in it Eqs. 3 and 4 gives the second equation of the model in a low frequency approximation $\left(\frac{\partial^{2}}{\partial t^{2}} \ll \omega_{p e}^{2}\right)$.

$\frac{\partial E_{z}}{\partial z}+E_{z} \frac{\partial \ln \varphi^{2} n}{\partial z}=-\frac{c^{2}}{\varphi v_{A}^{2} \omega_{p e}^{2}} \frac{\partial^{2}}{\partial t^{2}}\left(\frac{\partial E_{x}}{\partial x}+\frac{\partial \ln n}{\partial x} E_{x}\right)$

Eq. 5 is adapted to situations corresponding to the existence of low frequency fluctuations that drive the plasma dynamics. When LF waves (like Alfvén waves which are the source of the perturbation we focus on) no longer exist in the medium one has to take the high frequency oscillations (plasma waves in the present low $\beta$ case) into account. However since we expect parallel electric field to develop only where $E_{x} \neq 0$, we will here only concentrate on the system of Eq. 2 and Eq. 5. Combining these two equations leads to a fourth order partial derivative equation:

$\frac{\partial^{2} E_{x}}{\partial t^{2}}-\frac{v_{A}^{2}}{\varphi} \frac{\partial^{2} \varphi E_{x}}{\partial z^{2}}=\frac{c^{2}}{\varphi^{2} \omega_{p e}^{2}} \frac{\partial^{2}}{\partial t^{2}}\left(\frac{\partial^{2} E_{x}}{\partial x^{2}}+\frac{\partial \ln n}{\partial x} \frac{\partial E_{x}}{\partial x}+\frac{\partial^{2} \ln n}{\partial x^{2}} E_{x}\right)$

The two components of the electric field are coupled through the last term of the right hand side. In fact, when the variation of $n$ along the field lines is negligible these components are decoupled and one obtains a single propagation equation for $E_{x}$. Such an approximation is valid for high altitudes where the density can be considered homogeneous in the $z$ direction, but as we want to study processes that extend down to the ionosphere we keep all terms in the following treatment. The LHS of Eq. 6 describes the 
propagation along magnetic field lines, whereas the RHS is linked to dispersive effects.

\section{A model of the auroral cavities}

For realistic models of auroral cavities, the strong inhomogeneities in both the $x$ and $z$ directions preclude the use of a Fourier analysis as well as a WKB approach. The system of Eqs. 2 and 5 has thus to be solved numerically. Schematically, given $E_{x}$ and $E_{z}$ at time $t$ and everywhere in (x,z), Eq. 6 (or Eq. 2) allows to compute $E_{x}$ at the next time step; $E_{z}$ is then obtained from Eq. 5. The vertical density profile is derived from a fit to measurements ( [Kletzing, 1994] and references therein). We choose a simple analytical expression for describing the perpendicular variation of the density linked to the presence of a cavity: $n(x)=$ $n_{0}\left(1-\left(\arctan \left(\frac{x-L_{x} / 3}{\Delta_{g}}\right)+\arctan \left(\frac{-x+2 L_{x} / 3}{\Delta_{g}}\right)\right) / \pi\right)$. $\Delta_{g}$, the key parameter of the model, has values compatible with observations. It typically corresponds to variations of the density by a factor of 5 over 1 $\mathrm{km}$ (see [Strangeway et al., 1998]). The vertical structure of the auroral cavity is not known precisely. Deep plasma cavities of limited perpendicular extension have been crossed by Viking, Freja and FAST over the altitude range $[2000 \mathrm{~km}, 12000 \mathrm{~km}]$. Thus, we will assume that the cavity extends almost down to the ionosphere. Conversely the plasma is homogeneous at high altitudes (over $18000 \mathrm{~km}$ ). A typical Alfvén velocity profile used in the simulation is given in Fig. 1. The density in the cavity here is twice smaller than in the external plasma. Nevertheless, a limit of $50 \mathrm{~cm}^{-3}$ is taken for the maximum depletion.

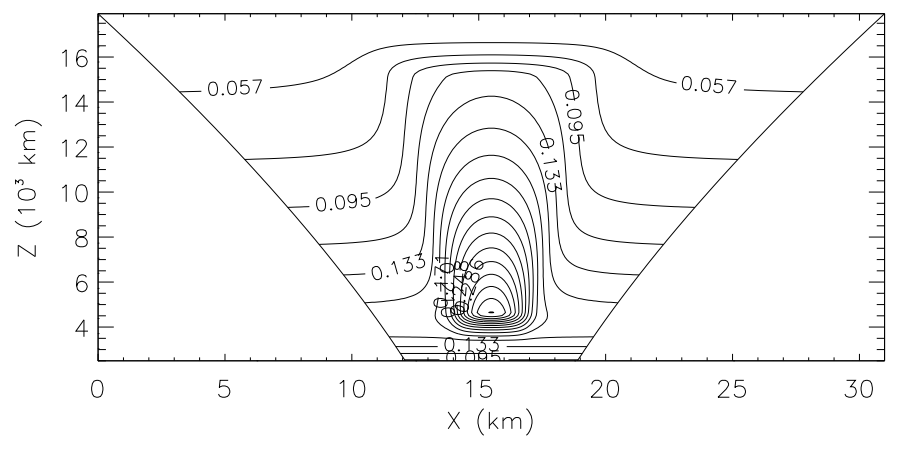

(this is taken into account in the different views of the simulation box). The magnetic field variation and the vertical density profile are typical of the ones observed at $70^{\circ}$ of latitude. The simulation is initialized by assuming that a pulse of arbitrary $z$ shape and homogeneous in $x$ enters the top of the box at the Alfvén velocity. The corresponding parallel electric field is then zero. Nevertheless, to gain generality, it is possible to initialize the field with a small $k_{\perp}$ without changing the numerical methods. The assumption of initially homogeneous pulse in the $x$ direction is just a question of simplification of the discussion. One of the goals of the study is to estimate the distance over which the pulse acquires small perpendicular wavelengths. This is easier to achieve using pure $k_{\|}$pulse. At the other side of the calculation box, we use a total reflection model for the ionosphere $\left(E_{\perp}=0\right)$ which is justified by the high conductivity of this medium. On both side of the box $\left(x=0\right.$ and $\left.x=L_{x}\right)$ there is no gradient, the plasma is homogeneous $\left(\frac{\partial}{\partial x}=0\right)$ and the field $\left(E_{\perp}, E_{\|}=0\right)$ obeys a typical wave equation which can be solved independently given the velocity variation along the field lines. Inside the simulation box we use numerical schemes for boundary value problems. In the $x$ direction, using finite differences, the solution is determined everywhere on the interval of interest, $\left[0, L_{x}\right]$, by solving a matrix equation (the so-called box method, see [Zwillinger, 1992]). In the $z$ direction a finite difference method with boundary and initial conditions for a hyperbolic wave equations is implemented.

\section{Propagation of Alfvén pulses}

The propagation of a pulse having an initial Gaussian shape and an amplitude of $E_{0} \sim 135 \mathrm{mV} / \mathrm{m}$ (at the top of the box $): E_{\perp}=E_{0} \exp \left(-\left(\frac{L_{z}-z+v_{A} t+z_{\infty}}{\Delta_{l}}\right)^{2}\right)$ is described in Fig. 2 (given the symmetry in $x$, one half of the simulation box is displayed). $\Delta_{l}$ is such that the pulse propagating at the Alfvén velocity would correspond to a temporal perturbation having a $1 \mathrm{~Hz}$ dominant frequency. One has taken $\Delta_{g}=0.5$ which corresponds to a moderate cavity. Three maps of the perpendicular electric field are presented for $t=1300,1600,1900 \omega_{c i}^{-1}$ (here $\omega_{c i}^{-1} \simeq 0.33 \mathrm{~ms}$ ) and

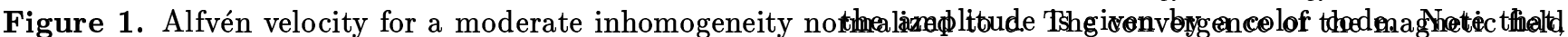

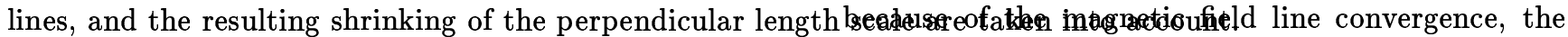

The simulation box extends from $2000 \mathrm{~km}$ up to $20000 \mathrm{~km}$. Due to the convergence of the magnetic field lines, the width of the box (North/South direction) is $30 \mathrm{~km}$ at $20000 \mathrm{~km}$ and $6 \mathrm{~km}$ at $2000 \mathrm{~km}$ $E_{\perp}$ values vary towards the Earth with a factor proportional to $\varphi^{-1}$. This implies an enhancement of a factor of approximately 5 between the upper and the lower sides of the box. 

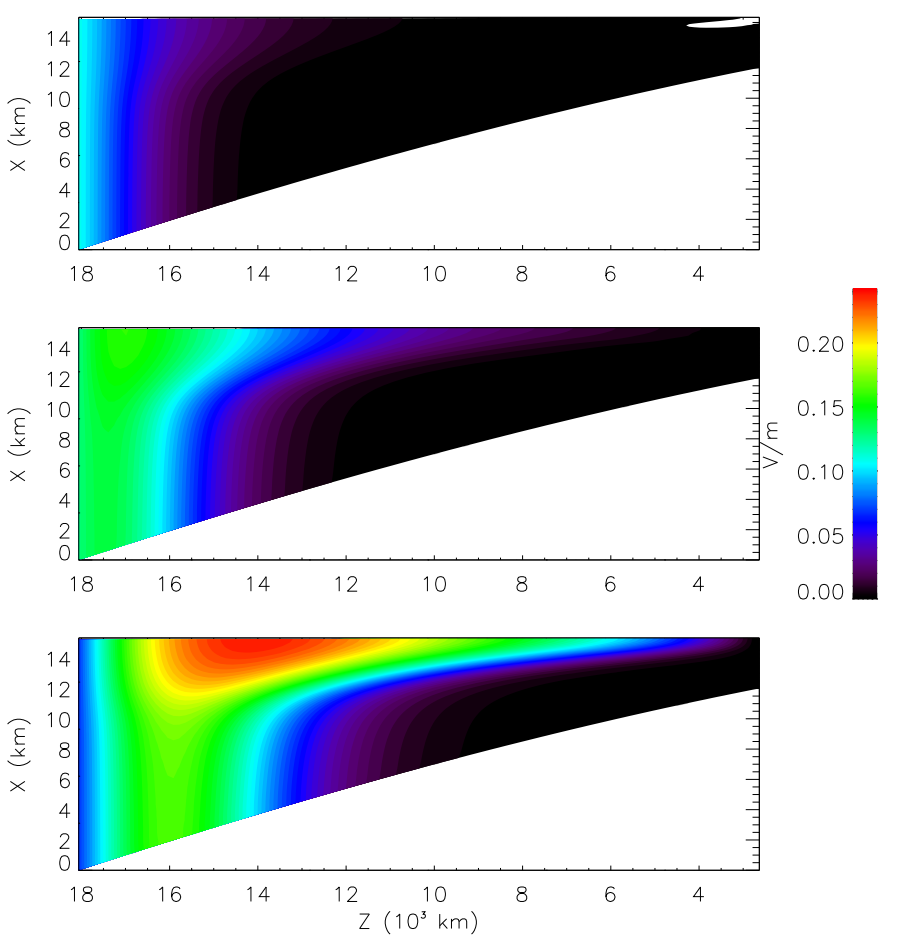

Figure 2. Perpendicular component of the electric field for and $1900 \omega_{c i}^{-1}$.

At the highest altitudes, no transverse effects distort the pulse which therefore remains homogeneous. As the pulse penetrates the cavity, it undergoes a transverse distortion due to the transverse variation of the Alfvén velocity. The perpendicular wavelength shrinks down to length of the order of the electron inertial length. In this last map, the equivalent $k_{\perp}^{-1}$ is of the order of $c / \omega_{p e}$ over the altitude range $[9000 \mathrm{~km}$, $14000 \mathrm{~km}$ ], in the regions of maximum gradient. The important point shown here is that a cavity comparable to the ones actually observed is able to create the small scales mandatory for the creation of a significant parallel electric field even after a short propagation (less than $5000 \mathrm{~km}$, approximately one wavelength) of the AW.

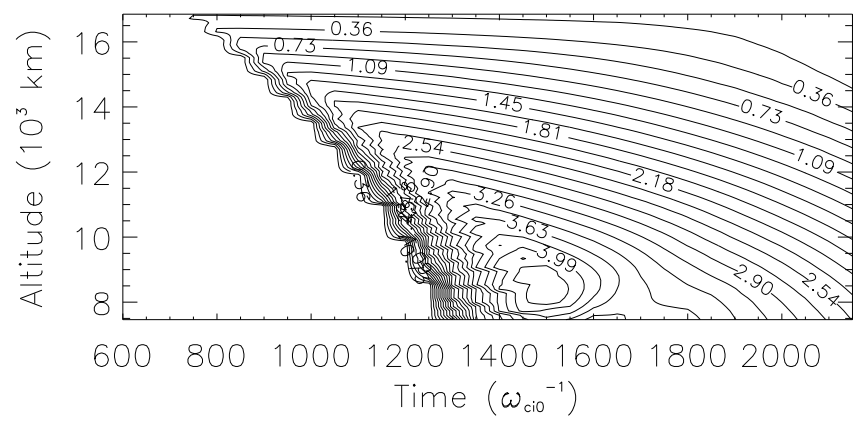

Figure 3. Maximum of the ratio $\frac{\delta_{e}}{E_{\perp}} \cdot \frac{\partial E_{\perp}}{\partial x}$ along the $x$ direction.

This is studied in a more general way in Fig. 3 where the temporal evolution of the typical perpendicular scale of the electric field is displayed. We particularly focused on the range of altitude where this scale minimizes. To build this figure, the ratio between $c / \omega_{p e}$ and the typical perpendicular inhomogeneity scale: $\eta=\frac{\delta_{e}}{E_{\perp}} \cdot \frac{\partial E_{\perp}}{\partial x}$ is calculated in the $(x, z)$ domain at each time step. Then, for any given time and altitude, we retain the maximum value of this ratio that is observed where the gradient maximizes. Starting from very low values (corresponding to ho mogeneous case), $\eta$ reaches values close $e^{i}$ to 1 after ${ }^{i} a$ propagation of only $2000 \mathrm{~km}$ inside the cavity. Moreover $\eta$ can be $\geq 1$ over a significantly extended altitude range (several thousands $\mathrm{km}$ at $t \sim 1500 \omega_{c i}^{-1}$ ) denoting the inertial regime of the wave. The $z$ dependence of the density tends to reduce the value of $\eta$ in the Earth direction.

Auroral density cavities are then sufficiently deep for considerably distorting the wave front. Whatever its initial shape, an AW pulse entering an auroral density cavity can thus quickly gain the small transverse length scales compatible with the formation of a significant parallel electric field.

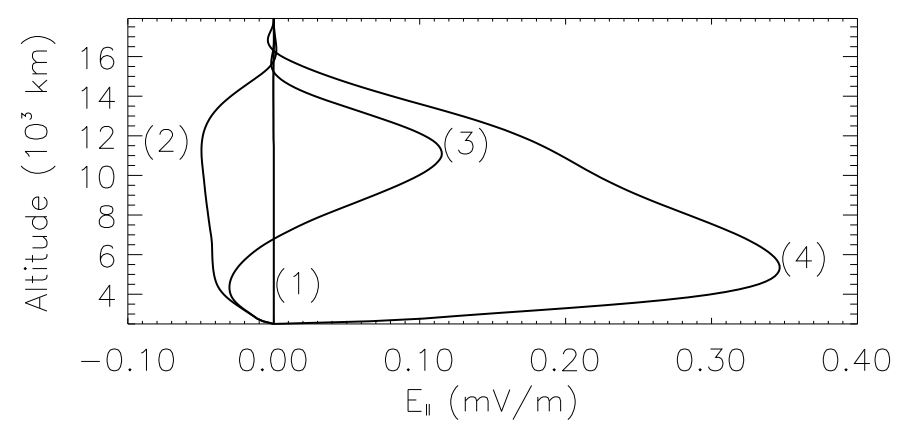




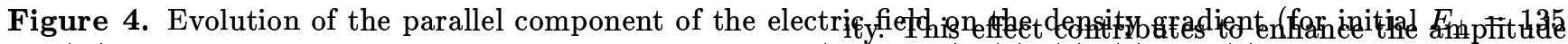

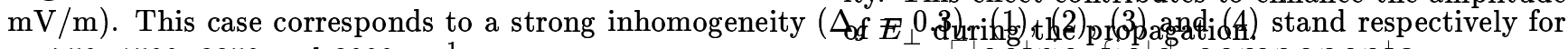
$t=750,1500,2250$ and $3000 \omega_{c i}^{-1}$.

How strong can this parallel field be? An example is given in Fig. $4\left(\Delta_{g}=0.3\right)$ where the temporal evolution of $E_{\|}$is displayed for $x$ corresponding to the locus of maximum gradient value. $E_{\|} / E_{\perp}$ values of the order of $1 / 400$ are reached. As seen on Fig. $4 E_{\|}$ can form over altitude ranges of the order of 10000 $\mathrm{km}$. Initial perturbations of a few $100 \mathrm{mV} / \mathrm{m}$ which are actually observed by Interball or Polar, for instance, would thus lead to a total potential drop of a few $\mathrm{keV}$. Outside of the density gradient $E_{\|}$is almost zero as we can see on Fig. 5 that we discuss later on. It is important to note that the integration of Eq. 5 for obtaining the parallel electric field is only justified in the region where the perpendicular electric field associated to the propagation of the AW is present. This equation indeed leads to a constant $E_{\|}$where $E_{\perp}=0$, which is clearly not physical. As mentioned before, the LF approximation used to derive the equations of the model is no longer valid in the region where $E_{\perp} \sim 0$. A detailed calculation (out of the scope of the present study) shows that the wave response in that region is then simply the plasma eigenmode (oscillations at $\omega_{p e}$ ). $E_{\|}$presents a complex spatio-temporal evolution, its sign and its amplitude being related to both the ones of $\partial \ln n / \partial x$ and of $\partial E_{\perp} / \partial z$. Therefore, the initial shape of the pulse is important in order to form significant parallel electric field.

To get a better understanding of the spatio-temporal coupling between the components of the electric field, Fig. 5 shows both $E_{\|}$and $E_{\perp}$ superimposed (it should be noted that the scales are different for the two components). Several facts can be noticed. First $E_{\|}$ reaches the higher values exactly where the wavefront of $E_{\perp}$ is the more distorted (ie where $k_{\perp} \neq 0$ ). This is also the locus of the density gradient in the $(x, z)$ plane. In the present case, the integration of those values $(\geq 0.06 \mathrm{mV} / \mathrm{m})$ on the region where they exist $(\sim 8000 \mathrm{~km})$ gives a potential drop of $0.5 \mathrm{kV}$ (note that the maximum value of $E_{\perp}$ is only $0.15 \mathrm{~V} / \mathrm{m}$ on the gradient). As already quoted much higher $E_{\perp}$ values are observed suggesting that this effect of wave distortion on the gradient can lead to the formation of significant potential drop of a few $\mathrm{keV}$. Outside of the gradient the pulse propagates without distortion, no $E_{\|}$is formed. In that case the shape modification of the pulse is due to the magnetic field line convergence and to the longitudinal variation of the Alfvén veloc-

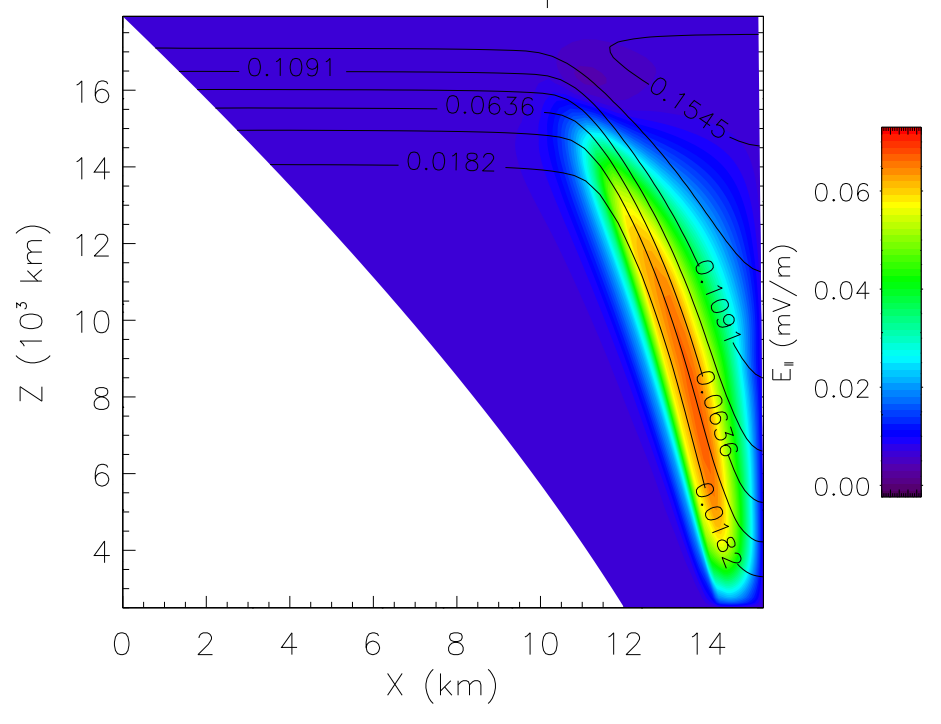

Figure 5. The two components of the electric field at a given perpendicular (line contours, in $\mathrm{V} / \mathrm{m}$ ).

Finally let us note that the ionospheric boundary conditions we chose (infinite conductivity) play a role in the formation of larger $E_{\|}$. The (total) reflection at the ionosphere induces an enhancement of the value of $E_{\|}$because of the differential propagation velocity. The center of the pulse travels faster that the sides, and thus will reflect sooner. After the reflection of the center, but before the one of the sides of the pulse, the inversion of the direction of propagation increases the $x$ derivative of $E_{\perp}$ and consequently the value of $E_{\|}$. This effect is indeed observed in the simulations but it may not appear with realistic boundary conditions.

As sketched in Fig. 6, the maximum of the parallel field varies almost like the inverse of the inhomogeneity scale $\Delta_{g}$. This variation allows strong parallel fields to be built with values of density gradients compatible with the observations $\left(E_{\|} / E_{\perp}\right.$ of the order of up to $1 / 150$ for an inhomogeneity parameter $\Delta_{g}=0.1$ that corresponds to a length scale of a few $100 \mathrm{~m}$ in the perpendicular direction at $3000 \mathrm{~km}$ ). This enables potential drops of a few $\mathrm{kV}$ to form which are typically the required values for explaining the observed particle energies. 


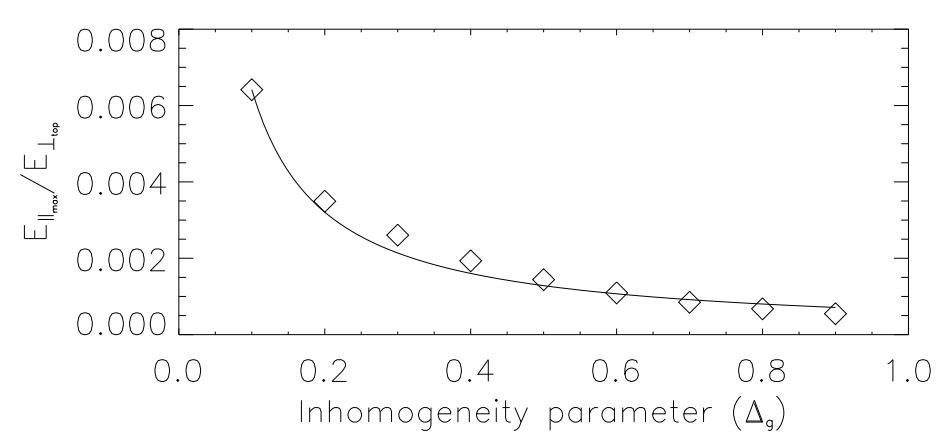

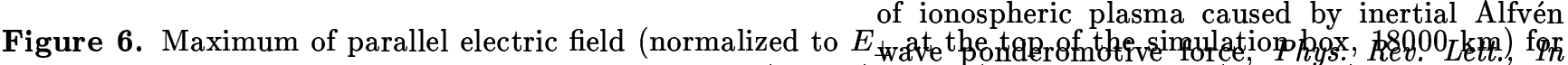

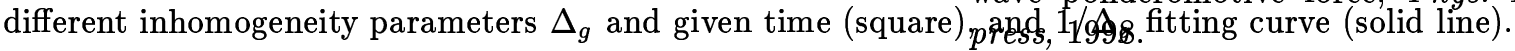

These values of $E_{\|}$, obtained on the gradient, are significantly larger than generally reported in the literature concerning parallel fields linked to Alfvén waves. Indeed, in the absence of density gradients, with only the contribution of the perpendicular wave vector of the electric field for inducing an electric space charge, the value of the potential drop would remain roughly ten times smaller.

A simple calculation can help to estimate the wave/particle energy transfer rate of the AW. The acceleration regions crossed by Viking correspond to current structures with associated current densities $j_{\|}$ of the order of a few $\mu \mathrm{A} / \mathrm{m}^{2}$. The dissipation linked to the formation of a parallel field of $1 \mathrm{mV} / \mathrm{m}$ would thus be typically of a few $10^{-9} \mathrm{~W} / \mathrm{m}^{3}$. This corresponds to the dissipation of the incident Poynting flux of the Alfvén waves (a few $10^{-3} \mathrm{~W} / \mathrm{m}^{2}$ ) over a distance of $1000 \mathrm{~km}$ which is of the order of one wavelength. Of course, the real situation inside the acceleration regions is much more complicated than what is described by our simple bi-fluid model and, for example, $j_{\|} \cdot E_{\|}$does not necessarily correspond to a dissipation (the direction of $E_{\|}$depends on the orientation of $E_{\perp}$ and of the density gradients). Nevertheless, our study suggests that particular small scale structures presenting simultaneously features of plasma cavities and current sheets are possible sites for the formation of large parallel electric fields.

\section{Conclusion}

Thanks to a realistic model of the auroral density cavities, we have shown that, on density gradients locus, it is possible to build parallel electric fields with amplitude and vertical extension sufficient to accelerate particles up to auroral energies. Typically, an Alfvénic perturbation having an amplitude and a frequency similar to the ones observed (a few $100 \mathrm{mV} / \mathrm{m}$ at $1 \mathrm{~Hz}$ ), entering the type of plasma cavities that have been crossed regularly by Viking and FAST, would develop a parallel electric field of the order of $1 \mathrm{mV} / \mathrm{m}$. Undoubtly, such amplitudes lead to an important energy dissipation and non-linear effects. They are not taken into account in the present linear study and are reserved for future works.

\section{References}

Bellan, P.M., and K. Stasiewicz, Fine-scale cavitation

Borovsky, J. E., Auroral arc thicknesses as predicted by various theories, J. Geophys. Res., 98, 6101, 1993.

Ergun, R. E., C.W. Carlson, J.P. McFadden, F.S. Mozer, G.T. Delory, W. Peria, C.C. Chaston, M. Temerin, R. Elphic, R. Strangeway, R. Pfaff, C.A. Cattell, D. Klumpar, E. Shelly, W. Peterson, E. Moebius, and L. Kistler, Fast satellite wave observations in the AKR source region, Geophys. Res. Lett., 25, 2061, 1998.

Goertz, C. K., and R. W. Boswell, Magnetosphereionosphere coupling, J. Geophys. Res., 84, 7239, 1979 .

Hasegawa, A., Particle acceleration by MHD surface waves and formation of aurora, J. Geophys. Res., $81,5083,1976$.

Hilgers, A., B. Holback, G. Holmgren, and R. Boström, Probe measurements of low plasma densities with application to the auroral acceleration regions and auroral kilometric radiation sources, $J$. Geophys. Res., 97, 8631, 1992.

Kletzing, C. A., Electron acceleration by kinetic Alfvén waves, J. Geophys. Res., 99, 11095, 1994.

Louarn P., A. Roux, H. de Féraudy, D. Le Quéau, M. André, and L. Matson, Trapped electrons as a free energy source for the auroral kilometric radiation, J. Geophys. Res., 95, 5983, 1990.

Louarn P., J.E. Wahlund, T. Chust, H. de Féraudy, A. Roux, B. Holback, P.O. Dovner, A.I. Eriksson, and G. Holmgren, Observation of kinetic Alfvén waves by the Freja spacecraft, Geophys. Res. Lett., 21, 1847, 1994.

Lysak, R.L., Electrodynamics coupling of the magnetosphere and ionosphere, Space Sci. Rev., 52, 33, 1990. 
Maggs, J.E., and G.J. Morales, Magnetic fluctuations associated with field-aligned striations, Geophys. Res. Lett., 23, 633, 1996.

Maggs, J.E., and G.J. Morales, Fluctuations associated with a filamentary density depletion, Phys. Plasmas, 4, 2, 1997.

Seyler, C.E., J.E. Wahlund and B. Holback, Theory and simulation of low-frequency plasma waves and comparison to Freja satellite observations, J. Geophys. Res., 100, 21453, 1995.

Stasiewicz, K., G. Holmgren, L. Zanetti, Density depletions and current singularities observed by Freja, J. Geophys. Res., 103, 4251, 1998.

Strangeway, R.J., L. Kepko, R.C. Elphic, C.W. Carlson, R.E. Ergun, J.P. McFadden, W.J. Peria, G.T. Delory, C.C. Chaston, M. Temerin, C.A. Cattell, E. Moebius, L.M. Kistler, D.M. Klumpar, W.K. Peterson, E.G. Shelley, and R.F. Pfaff, Fast observations of VLF waves in the auroral zone: Evidence of very low plasma densities, Geophys. Res. Lett., 25, 2065, 1998.

Streltsov, A.V, and W. Lotko, Dispersive, nonradiative field line resonances in a dipolar magnetic field geometry, J. Geophys. Res., 102, 27121, 1997.

Wahlund, J. E., Louarn, P., T. Chust, H. de Féraudy, A. Roux, B. Holback, P.O. Dovner and G. Holmgren, On ion-acoustic turbulence and the nonlinear evolution of kinetic Alfvén waves, Geophys. Res. Lett., 21, 1831, 1994.

Volwerk, M., P. Louarn, T. Chust, A. Roux, H. de Féraudy, and B. Holback, Solitary kinetic Alfvén waves: A study of the Poynting flux, J. Geophys. Res., 101, 13335, 1996.

Zwillinger, D., Handbook of differential equations, Academic Press, 1992.

V. Génot, P. Louarn ${ }^{1}$ Observatoire Midi-Pyrénées, 14 Av. Edouard Belin, 31400 Toulouse, France. (email: genot@obs-mip.fr, louarn@obs-mip.fr)

D. Le Quéau, Centre d'Etude Spatiale des Rayonnements, 9 Av. Colonel Roche, 31400 Toulouse, France. (e-mail: lequeau@cesr.fr)

23 July 1998; revised 17 March 1999; accepted 18 March 1999.

\footnotetext{
${ }^{1}$ Now both at CESR.

This preprint was prepared with AGU's $\mathrm{L}_{\mathrm{A}} \mathrm{T} \mathrm{X}$ macros v4. File prep formatted January 18, 2001.
} 